\section{OPEN ACCESS}

Edited and reviewed by: Wenliang Song,

Vanderbilt University Medical Center,

United States

${ }^{*}$ Correspondence:

Wei Wang

wangwei_sir@126.com

Zheng-Zhu Zhang

zzz@ahau.edu.cn

Li-Long Pan

Ilpan@jiangnan.edu.cn

Specialty section:

This article was submitted to Inflammation Pharmacology,

a section of the journal

Frontiers in Pharmacology

Received: 22 March 2018

Accepted: 18 April 2018

Published: 01 May 2018

Citation:

Wang W, Zhang Z-Z, Wu Y

Wang R-Q, Chen J-W, Chen J,

Zhang Y, Chen Y-J, Geng M, Xu Z-D,

Dai M, Li J-H and Pan L-L (2018)

Corrigendum:

(-)-Epigallocatechin-3-Gallate

Ameliorates Atherosclerosis and Modulates Hepatic Lipid Metabolic Gene Expression in Apolipoprotein E

Knockout Mice: Involvement of

TTC39B Front. Pharmacol. 9:459.

doi: 10.3389/fphar.2018.00459

\title{
Corrigendum: (-)-Epigallocatechin-3- Gallate Ameliorates Atherosclerosis and Modulates Hepatic Lipid Metabolic Gene Expression in Apolipoprotein E Knockout Mice: Involvement of TTC39B
}

\begin{abstract}
Wei Wang ${ }^{1 *}$, Zheng-Zhu Zhang ${ }^{2 *}$, Yan Wu ${ }^{1}$, Ru-Qing Wang ${ }^{1}$, Jin-Wu Chen ${ }^{1}$, Jing Chen ${ }^{1}$, Yan Zhang ${ }^{1}$, Ya-Jun Chen ${ }^{1}$, Ming Geng ${ }^{1}$, Zhong-Dong Xu ${ }^{1}$, Min Dai ${ }^{3}$, Jin-Hua $\mathrm{Li}^{1}$ and Li-Long Pan ${ }^{4 *}$
\end{abstract}

${ }^{1}$ School of Life Science, Hefei Normal University, Hefei, China, ${ }^{2}$ State Key Laboratory of Tea Plant Biology and Utilization, Anhui Agricultural University, Hefei, China, ${ }^{3}$ Key Laboratory of Xin'an Medicine, Ministry of Education, Anhui Key Laboratory for Research and Development of Traditional Chinese Medicine, School of Pharmacy, Anhui University of Chinese Medicine, Hefei, China, ${ }^{4}$ School of Medicine, Jiangnan University, Wuxi, China

Keywords: (-)-Epigallocatechin-3-gallate, tetratricopeptide repeat domain protein 39B, atherosclerosis, inflammation, dyslipidemia

\section{A corrigendum on}

(-)-Epigallocatechin-3-Gallate Ameliorates Atherosclerosis and Modulates Hepatic Lipid Metabolic Gene Expression in Apolipoprotein E Knockout Mice: Involvement of TTC39B by Pan, L., Wu, Y., Wang, R., Chen, J., Chen, J., Zhang, Y., et al. (2018). Front. Pharmacol. 9:195. doi: 10.3389/fphar.2018.00195

The authorship of this article should be correctly ordered to protect the right ownership of this paper. In addition, Dr. Li-Long Pan has contributed significantly to this study, such that Dr. Pan should be the co-corresponding author in this study. The authorship order should be considered as follows:

Wei Wang ${ }^{1 *}$, Zheng-Zhu Zhang ${ }^{2 *}$, Yan $\mathrm{Wu}^{1}$, Ru-Qing Wang ${ }^{1}$, Jin-Wu Chen ${ }^{1}$, Jing $\mathrm{Chen}^{1}$, Yan Zhang ${ }^{1}$, Ya-Jun Chen ${ }^{1}$, Ming Geng ${ }^{1}$, Zhong-Dong $\mathrm{Xu}^{1}$, Min Dai ${ }^{3}$, Jin-Hua $\mathrm{Li}^{1}$ and Li-Long Pan ${ }^{4 *}$

${ }^{1}$ School of Life Science, Hefei Normal University, Hefei, China.

${ }^{2}$ State Key Laboratory of Tea Plant Biology and Utilization, Anhui Agricultural University, Hefei, China.

${ }^{3}$ Key Laboratory of Xin'an Medicine, Ministry of Education, Anhui Key Laboratory for Research and Development of Traditional Chinese Medicine, School of Pharmacy, Anhui University of Chinese Medicine, Hefei, China.

${ }^{4}$ School of Medicine, Jiangnan University, Wuxi, China. 
The authors apologize for this error and state that this does not change the scientific conclusions of the article in any way. The original article has been updated.

\section{AUTHOR CONTRIBUTIONS}

WW and Z-ZZ designed the experiments. WW, YW, R-QW, JC, J-WC, YZ, Y-JC, MG, Z-DX, and L-LP performed the experiments. WW, Z-ZZ, and L-LP performed the data analysis. $\mathrm{MD}, \mathrm{J}-\mathrm{HL}, \mathrm{WW}$, and L-LP wrote the manuscript.
Conflict of Interest Statement: The authors declare that the research was conducted in the absence of any commercial or financial relationships that could be construed as a potential conflict of interest.

Copyright (ㅇ 2018 Wang, Zhang, Wu, Wang, Chen, Chen, Zhang, Chen, Geng, $X u, D a i, L i$ and Pan. This is an open-access article distributed under the terms of the Creative Commons Attribution License (CC BY). The use, distribution or reproduction in other forums is permitted, provided the original author(s) and the copyright owner are credited and that the original publication in this journal is cited, in accordance with accepted academic practice. No use, distribution or reproduction is permitted which does not comply with these terms. 